\title{
Polarimetric evolution of V838 Monocerotis ${ }^{\star}$
}

\author{
S. Desidera ${ }^{1}$, E. Giro ${ }^{1}$, U. Munari ${ }^{2}$, Y. S. Efimov ${ }^{3}$, A. Henden ${ }^{4}$, S. Benetti ${ }^{1}$, T. Tomov ${ }^{5}$, \\ A. Bianchini ${ }^{6}$, and C. Pernechele ${ }^{1}$
}

1 INAF - Osservatorio Astronomico di Padova, Vicolo dell' Osservatorio 5, 35122, Padova, Italy

2 INAF - Osservatorio Astronomico di Padova - Sede di Asiago, 36012, Asiago, Italy

3 Crimean Astrophysical Observatory and Isaac Newton Institute of Chile, Crimean Branch, Ukraine

${ }^{4}$ Universities Space Research Association/US Naval Observatory Flagstaff Station, PO Box 1149, Flagstaff AZ 86002-1149, USA

5 Centre for Astronomy Nicholaus Copernicus University, ul. Gagarina 11, 87-100 Torun, Poland

${ }^{6}$ Universitá di Padova, Dipartimento di Astronomia, Vicolo dell'Osservatorio 2, 35122, Padova, Italy

Received 28 April 2003 / Accepted 30 September 2003

\begin{abstract}
We present the results of our polarimetric and spectropolarimetric monitoring of V838 Monocerotis, performed at the Asiago and Crimean observatories during and after the multiple outbursts that occurred in January-March 2002. The polarization of the object is mainly due to interstellar polarization $(P \sim 2.48 \%)$. Intrinsic polarization up to $\sim 0.7 \%$ at $5000 \AA$ is present during the second maximum of the object (February 2002). This intrinsic component increases toward shorter wavelengths but our limited spectral coverage (5000-7500 $\mathrm{A}$ ) does not allow conclusive inferences about its origin. A strong depolarization across the $\mathrm{H}_{\alpha}$ profile is observed. The interstellar polarization gives a lower limit to the reddening of $E(B-V)>0.28$, with $E(B-V) \sim 0.5$ being the most probable value. A normal ratio of total to selective absorption $\left(R_{V}=3.22 \pm 0.17\right)$ was derived from the wavelength of maximum interstellar polarization. This suggests a low (if any) contribution by circumstellar material with a peculiar dust to gas ratio. A polarimetric map of a portion of the light echo shows a complex polarization distribution reaching $P_{\max }=45 \%$.
\end{abstract}

Key words. stars: individual: V838Mon - techniques: polarimetric - stars: peculiar (except chemically peculiar)

\section{Introduction}

V838 Monocerotis developed a spectacular multiple outburst in January-March 2002, reaching $V=6.7$. Its spectral characteristics changed dramatically during its evolution. The progenitor had the temperature of a F star, while during the outburst V838 Mon evolved from a cool K giant to a late M giant. The profile of spectral lines also changed during the outburst, and a strong $\mathrm{H}_{\alpha}$ emission appeared during the second maximum, when the object reached its peak visual magnitude. A prominent light echo was discovered by Henden et al. (2002). The evolution of V838 Mon from January to April 2002 is described by Munari et al. (2002a). Further photometric and spectroscopic observations were presented by Kimeswenger et al. (2002), Goranskii et al. (2002), Kolev et al. (2002), Banerjee \& Ashok (2002), Wisniewski et al. (2003), and Crause et al. (2003). The first spectra obtained after the emersion from solar conjunction revealed a dramatic temperature decrease of the object, whose spectrum became dominated by $\mathrm{TiO}$ and VO molecular bands, suggesting a spectral type later than

Send offprint requests to: S. Desidera,

e-mail: desidera@pd.astro.it

* Based on observations collected at Padua Astronomical Observatory at Asiago and Crimean Astrophysical Observatory.
M10-III (Desidera \& Munari 2002). A faint blue continuum was found to dominate the spectrum blueward of $7000 \AA$, indicating a likely binary nature for the object. The hot component was classified as B3V by Munari et al. (2002b). Bond et al. (2003) studied the light echo using the ACS onboard the HST. They found a lower limit to the distance of $6 \mathrm{kpc}$, implying that V838 Mon at its maximum brightness was temporarily the brightest star in the Milky Way.

In spite of these observational efforts the nature of V838 Mon remains largely unknown.

The study of the polarization can shed light on some physical properties. On the one hand the interstellar polarization gives clues about the distance and the absorption towards the object. On the other hand, the presence of intrinsic polarization, its wavelength dependence, its variations during the evolution of the object and the polarization across line profiles provide clues about the physics of the object. Wisniewski et al. (2003) present 2-epoch spectropolarimetric observations of V838 Mon. They reveal the presence of intrinsic polarization during the outburst, with variations across the profile of emission lines.

Here we present the results of our more extensive polarimetric and spectro-polarimetric monitoring of 
V838 Monocerotis, performed at the Asiago and Crimean observatories from January to November 2002. The results presented here supersede the premininary analysis of part of the same dataset included in Munari et al. (2002a).

\section{Observations}

\subsection{Asiago}

Polarimetric and spectropolarimetric observations of V838 Monocerotis were performed using the polarimetric mode of AFOSC at the $1.82 \mathrm{~m}$ telescope at Asiago Observatory (Italy). The AFOSC instrument is described by Desidera et al. (2002). The polarimeter is presented in detail elsewhere (Pernechele et al. 2003) and early results based on its use were presented by Giro et al. (2003). Here we recall the main characteristics of the instrument. The AFOSC polarimeter consists of a double Wollaston prism which splits the incoming light into four polarized beams $\left(\right.$ at $0^{\circ}, 90^{\circ},-45^{\circ}$ and $45^{\circ}$ ) separated by 20 arcesc. These four beams are in principle sufficient to determine the first three elements of the Stokes vector, i.e. the intensity $I$ and the two linear polarization parameters $Q$ and $U$. The Wollaston can be housed in the filter wheel or in the grism wheel of AFOSC. In the first case, spectropolarimetry can be performed by inserting a grism in the grism wheel, and in the latter imaging or photo-polarimetry is obtained by inserting a filter in the filter wheel.

For the spectropolarimetry, we used three different grisms: Grism \#4, (wavelength range 4500-7800 $\AA$; resolution $4.3 \AA$ ipixel), Grism \#7, (4350-6550 ̊; $2.2 \AA /$ pixel), Grism \#8, (6250-8000 $\AA$; $1.8 \AA$ /pixel). In all cases a slit 2.5 arcsec wide and 18 arcsec long was used.

Our observational procedure includes spectra taken at position angles of $0^{\circ}$ and $90^{\circ}$, to properly eliminate the spurious effects introduced by the different behaviour of the grism for the two polarimetric states. Flat fields were taken at both slit position angles $\left(0^{\circ}\right.$ and $\left.90^{\circ}\right)$ to avoid spurious polarization effects due to screen reflections.

Observations were mostly performed in service mode as a target of opportunity, and this explains some inhomogeneities of the instrument set-up used and, in some cases, the lack of observations of standard stars.

The first spectropolarimetric observations were performed very early after the first maximum (January 10), and then we continued our monitoring covering the relevant phases of the evolution of this peculiar object for nearly 2 months. In particular, the maximum of visual magnitude (Feb. 2002) is well covered by our observations.

When the object became too faint for spectropolarimetry, we continued our monitoring in polarimetric imaging. Deep images obtained in $V$ band also allow a study of the polarization of the light echo and of stars in the direction of V838 Mon.

Table 1 presents the journal of observations.

\subsection{Crimea}

Linear polarimetry of V838 Mon was carried out on February 11, 15, 16, 18 and March 9, 2002 with the $125 \mathrm{~cm}$
Table 1. Journal of observations. The grism is reported for spectropolarimetric observations (\#4, \#7, \#8, see text for details) and filter for polarimetric photometry. A and $\mathrm{C}$ refer to Asiago and Crimean observations respectively.

\begin{tabular}{ccccc}
\hline \hline Target & Date & UT & Grism/Filter & Obs. \\
\hline V838 Mon & $10 / 01 / 2002$ & 2340 & 4 & $\mathrm{~A}$ \\
V838 Mon & $11 / 01 / 2002$ & 2310 & 4 & $\mathrm{~A}$ \\
$\beta$ Cas & $11 / 01 / 2002$ & 2310 & 4 & $\mathrm{~A}$ \\
V838 Mon & $04 / 02 / 2002$ & 2245 & $4,7,8$ & $\mathrm{~A}$ \\
$\beta$ Vir & $05 / 02 / 2002$ & 0210 & $4,7,8$ & $\mathrm{~A}$ \\
HD 93521 & $05 / 02 / 2002$ & 0340 & $4,7,8$ & $\mathrm{~A}$ \\
V838 Mon & $11 / 02 / 2002$ & 1840 & $U, B, V, R, I$ & $\mathrm{C}$ \\
HD 42807 & $11 / 02 / 2002$ & 1930 & 8 & $\mathrm{~A}$ \\
V838 Mon & $11 / 02 / 2002$ & 2110 & 8 & $\mathrm{~A}$ \\
V838 Mon & $15 / 02 / 2002$ & 2100 & $U, B, V, R, I$ & $\mathrm{C}$ \\
V838 Mon & $16 / 02 / 2002$ & 2010 & $U, B, V, R, I$ & $\mathrm{C}$ \\
V838 Mon & $18 / 02 / 2002$ & 2130 & $U, B, V, R, I$ & $\mathrm{C}$ \\
V838 Mon & $18 / 02 / 2002$ & 2150 & 4 & $\mathrm{~A}$ \\
$\beta$ Vir & $18 / 02 / 2002$ & 2355 & 4 & $\mathrm{~A}$ \\
V838 Mon & $04 / 03 / 2002$ & 2130 & 4 & $\mathrm{~A}$ \\
V838 Mon & $09 / 03 / 2002$ & 2000 & $U, B, V, R, I$ & $\mathrm{C}$ \\
V838 Mon & $09 / 03 / 2002$ & 2100 & 8 & $\mathrm{~A}$ \\
HD 114710 & $09 / 03 / 2002$ & 0205 & 8 & $\mathrm{~A}$ \\
V838 Mon & $20 / 03 / 2002$ & 1910 & $B, V, I$ & $\mathrm{~A}$ \\
HD 98421 & $20 / 03 / 2002$ & 2220 & $B, V, I$ & $\mathrm{~A}$ \\
HD 204847 & $29 / 10 / 2002$ & 1920 & $V$ & $\mathrm{~A}$ \\
HD 14069 & $30 / 10 / 2002$ & 0025 & $V$ & $\mathrm{~A}$ \\
V838 Mon & $30 / 10 / 2002$ & 0420 & $V$ & $\mathrm{~A}$ \\
HD 42807 & $09 / 11 / 2002$ & 0155 & $V$ & $\mathrm{~A}$ \\
HD 43384 & $09 / 11 / 2002$ & 0220 & $V$ & $\mathrm{~A}$ \\
V838 Mon & $09 / 11 / 2002$ & 0350 & $V$ & $\mathrm{~A}$ \\
\hline
\end{tabular}

reflector at the Crimean astrophysical observatory (Ukraine), using the computer controlled UBVRI Double Image Chopping Photopolarimeter, developed at the Helsinki University observatory by V. Piirola (Piirola 1973, 1988).

The instrument has two operational modes: photometric mode with elimination of the background close to the object, and two polarimetric modes to measure linear and/or circular polarization. In these modes the background is measured before and after each set of observations of the object. An important advantage is that the sky polarization is directly eliminated by using a plane parallel calcite plate as the polarizing beam splitter.

The measurements in different colors are performed simultaneously using 5 photomultipliers and dichroic filters to split the light into five spectral regions centered at $0.36,0.44$, $0.53,0.69$ and $0.83 \mu \mathrm{m}$ with $F W H M$ equal to $0.04,0.08$, $0.08,0.18$ and $0.12 \mu \mathrm{m}$ respectively. The instrumental system is quite similar to the Johnson $U B V$ and Cousins $R_{\mathrm{C}}, I_{\mathrm{C}}$. The efficiency is high because there is practically no internal absorption in the dichroic beam splitters.

All observations have been carried out in the linear polarization mode. In the course of polarimetric measurements the instrument retarder plate rotated with $22.5^{\circ}$ steps in front of a polarizer. One complete measurement consists of eight integrations in different orientations of the waveplate. The duration 
of one measurement with $10 \mathrm{~s}$ integration time for each beam takes about 3 min. All observations were made with 10 arcsec aperture, with the typical seeing of about 3 arcsec. However, due to large zenith angles $\left(>50^{\circ}\right)$ the photometric quality of the nights was not good. The number of exposures was adapted according to the sky conditions and the magnitude of the target.

\section{Data analysis}

\subsection{Asiago}

The reduction of spectropolarimetric data was performed in the standard way using IRAF ${ }^{1}$. The analysis was performed using our own IDL scripts and the POLMAP software package maintained by Starlink ${ }^{2}$.

A polarization measurement is based on two frames acquired at slit position angles equal to $0^{\circ}$ and $90^{\circ}$. Each image contains four spectra, relative to the four polarization directions $\left(0^{\circ}, 90^{\circ}, 45^{\circ}\right.$ and $\left.-45^{\circ}\right)$ produced by the Wollaston prism. If we label these spectra as $I_{\theta 1}(\lambda), I_{\theta 2}(\lambda), I_{\theta 3}(\lambda), I_{\theta 4}(\lambda)$, with $\theta=0^{\circ}$, $90^{\circ}$ the slit position angle, the two Stokes linear parameters can be computed as:

$$
\begin{array}{ll}
Q_{\theta}=\frac{I_{\theta 1}(\lambda)-I_{\theta 2}(\lambda)}{I_{\theta 1}(\lambda)+I_{\theta 2}(\lambda)} & U_{\theta}=\frac{I_{\theta 3}(\lambda)-I_{\theta 4}(\lambda)}{I_{\theta 3}(\lambda)+I_{\theta 4}(\lambda)} \\
Q=\frac{Q_{0}-Q_{90}}{2} & U=\frac{U_{0}-U_{90}}{2} .
\end{array}
$$

On Feb. 4, spectropolarimetric observations were obtained with all the grisms used in the monitoring. As shown in Fig. 1, the results of the three grisms in the overlap region agree quite well. We can therefore safely merge the results obtained with the different grisms on different nights.

For the polarimetric analysis we used IDL scripts developed by us for this purpose. After bias subtraction and flat field division, aperture photometry is performed for the four polarimetric channels, properly subtracting the sky contribution. The resulting Stokes parameters are calculated as above, but using only one of the adapter position angles $\left(0^{\circ}\right)$.

In building the polarimetric map of the light echo (Sect. 4.8) the calculation of the Stokes parameters was performed pixel by pixel in the bidimensional image, after a fine alignment of the four polarimetric channels. Sky subtraction was performed using the POLPACK package.

On some nights zero polarimetric standards were not acquired. However, the instrumental polarization resulting from the analysis of the available standards (including additional observations on dates different from those of V838 Mon observations) is fairly constant and always below $0.3 \%$. Continuous observations of the same standard at different telescope positions and airmasses show a dispersion at a $0.2 \%$ level, mainly due to the scattering of light at slit edges (Lorenzi 2003). The analysis of standard stars with known high polarization indicates that systematic errors in the position angle are below $2.0^{\circ}$.

\footnotetext{
${ }^{1}$ IRAF is distributed by the National Optical Observatory, which is operated by the Association of Universities for Research in Astronomy, Inc., under contract with the National Science Fundation.

2 www.starlink.rl.ac.uk
}

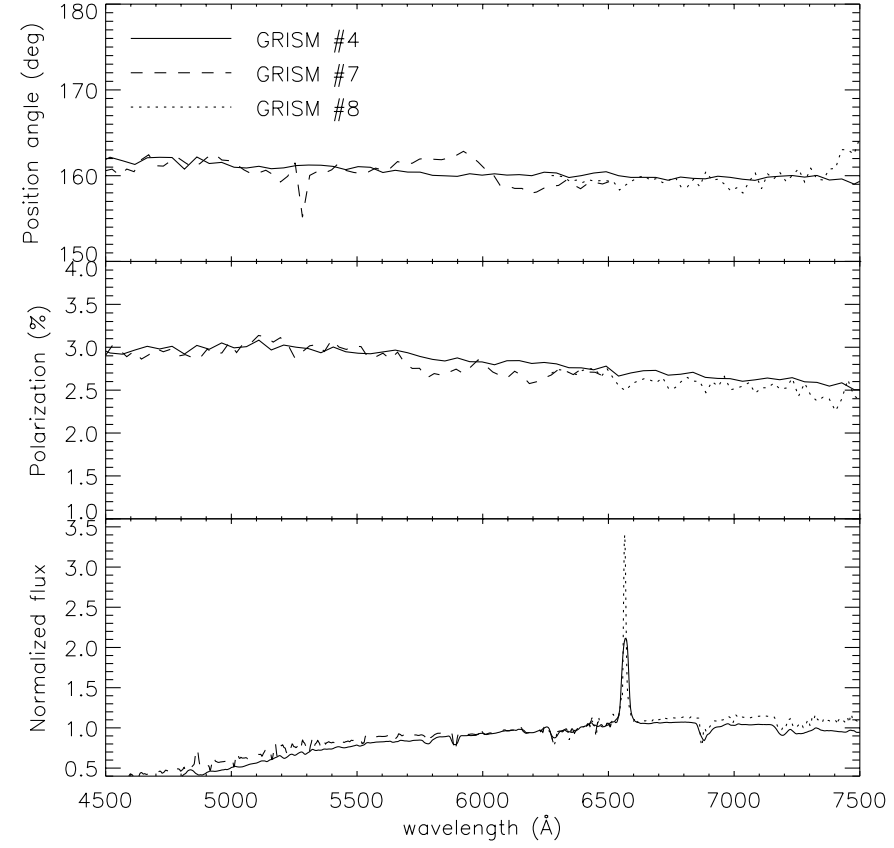

Fig. 1. Spectropolarimetry of V838 Mon on Feb. 4, 2002 using Grism \#4 (continuous line), Grism \#7 (dashed line) and Grism \#8 (dotted line). In the region of overlap, the results for the three grisms agree quite well. The peak intensity of $\mathrm{H}_{\alpha}$ emission obviously correlates with spectral resolution of different grisms. The feature in position angle at $\lambda \sim 5300 \AA$ is caused by a ghost.

\subsection{Crimea}

Before reduction all raw data are checked to eliminate bad points from the observations. The data reduction was carried out using the software package developed by Piirola, which performs all the required corrections (background subtraction, correction for the instrumental polarization, transformation into the equatorial system, transformation from instrumental magnitudes to the standard system, calculation of the weighted nightly means of the normalized Stokes parameters of polarization).

Polarimetric observations are corrected for instrumental polarization by applying the instrumental constants calculated for a number of polarimetric standards. The data were reduced with instrumental constants for the time interval of our observations (Feb.-Mar. 2002). Unfortunately, due to a problem during the realuminization process of the main mirror, the instrumental polarization is large and wavelength dependent, decreasing from $2.4 \%$ in $U$ to $0.5 \%$ in $I$ band. An inspection of the instrumental constant estimations obtained before and after the observations of V838 Mon (January-July 2002) has shown that their scattering does not exceed $0.1 \%$ in $U$ and is at the level of $0.02 \%$ in the other bands.

\section{Results}

\subsection{The polarization of V838 Mon}

The results of our measurement of polarization of V838 Mon are summarized in Table 2. To allow a comparison between the spectro-polarimetric results and the polarimetric photometry, 


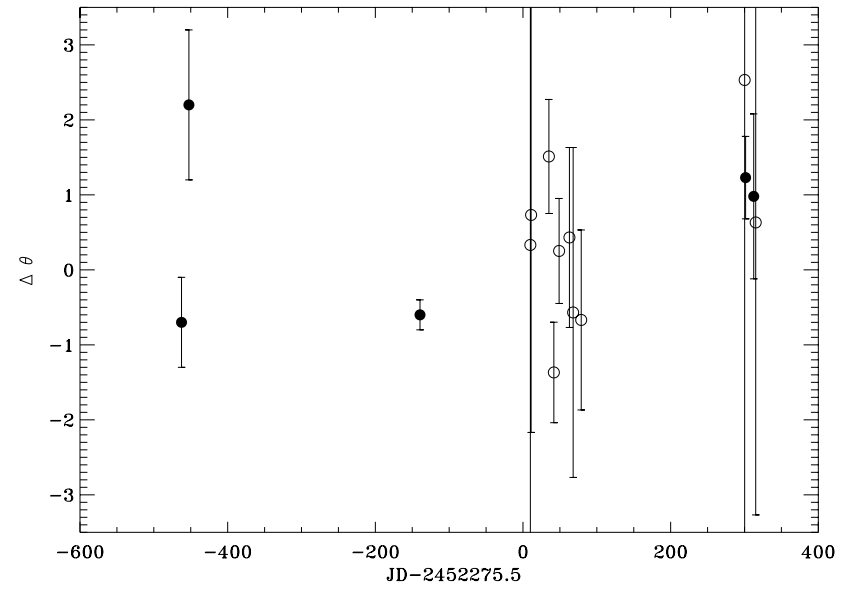

Fig. 2. Stability of position angle measurements with AFOSC polarimeter. Filled circles: difference between position angle of highpolarization standard stars measured by us and literature values. Empty circles: position angle measurement of V838 Mon taken with the AFOSC polarimeter (with the weighted average $159.1^{\circ}$ subtracted). Interstellar polarization dominates at all epochs, so that position angle of V838 Mon is expected to be nearly constant. The time coordinate is the same as Fig. 3. The results of standard stars leaves little room for systematic errors in position angle measurement with the AFOSC polarimeter exceeding $2^{\circ}$.

the integrated $V$ band polarization was measured on the spectra. Quoted errors of Asiago observations are those due to photon noise. Errors of Crimean data include photon noise and the error due to the scattering of the Stokes parameters between all 8 integrations of a complete single measurement. The Stokes parameters are weighted by the largest of these errors. The errors of the final data are calculated by the usual formula for weighted error.

The $V$ band polarization ranges between 2.35 and $3.09 \%$. The results of Asiago and Crimea observations agree fairly well, with differences at the $0.1 \%$ level. The wavelength range and effective wavelength of photometric bands depend on the spectral type of the object and may be significantly different from the standard ones for very red objects like V838 Mon. This could explain some systematic differences (i.e. polarization in $R_{\mathrm{C}}$ and $I_{\mathrm{C}}$ bands lower than the spectropolarimetric data, see Fig. 5). There is instead a position angle offset between the two datasets larger than the quoted errors. The position angle is about $159^{\circ}$ and $153^{\circ}$ for the Asiago and Crimea data, respectively.

We investigated in some detail the absolute calibration of position angle. Polarized standard stars were not observed at Asiago in January-March 2002; however the analysis of such standards taken at different epochs (including October and November 2002) reproduce the known position angles within $2^{\circ}$ (Fig. 2). A similar analysis performed on the Crimean polarimeter shows that typical systematic errors on position angles do not exceed 1.5. The discrepancy in position angle between Asiago and Crimea observations is larger than the corresponding uncertainties. In spite of our best efforts, no explanation has been obtained. However, this fact does not

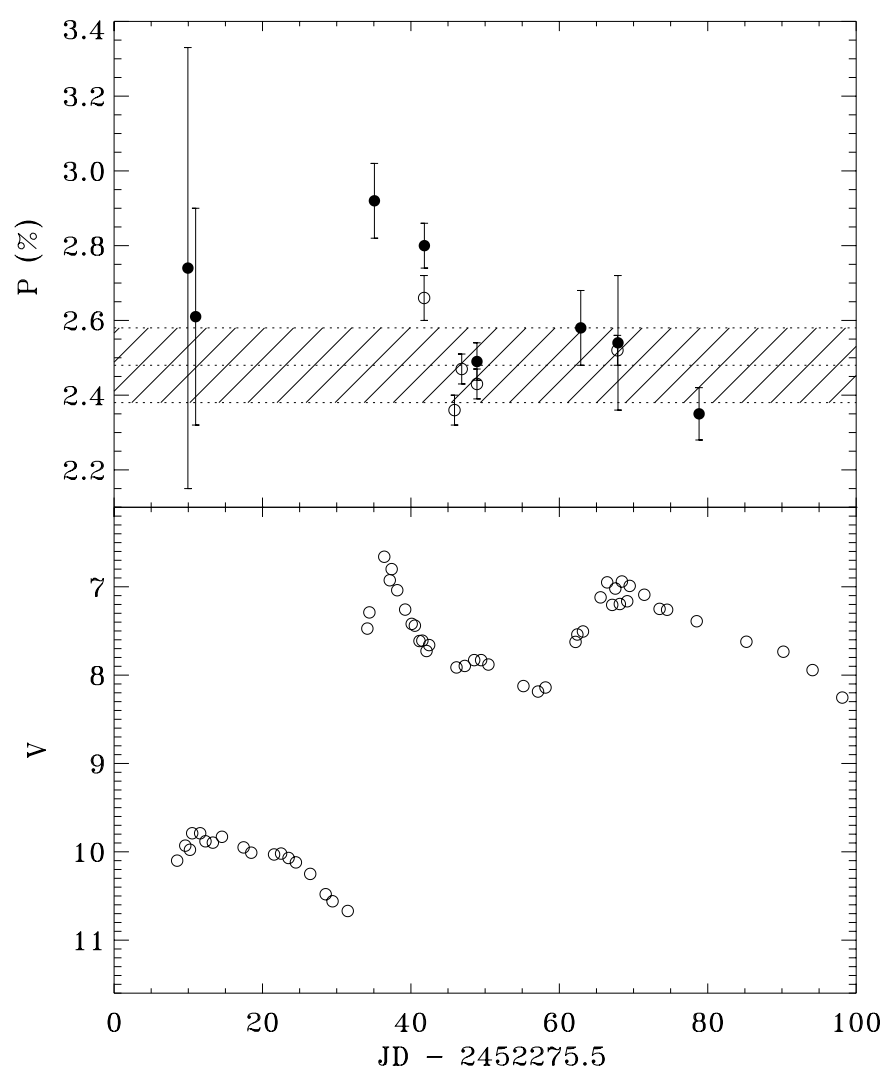

Fig. 3. Evolution of $V$ band polarization of V838 Monocerotis during the outburst (upper panel). Filled and empty circles represent the Asiago and Crimea data respectively. The shaded area shows our estimate of interstellar polarization ( $2.48 \pm 0.10 \%$ in $V$ band). The lower panel shows the photometric evolution of the object (from Munari et al. 2002a). The time coordinate is that used by Munari et al. (2002a). The intrinsic polarization appears correlated with the visual magnitude during the second and possibly the third maxima of the object. Two further polarization measurements at later epochs are outside of the plot limits.

significantly affect the main results of this study, since the absolute values of polarization agree very well.

The dependence of the polarization on wavelength is smooth and compatible with a Serkowski law (Serkowski et al. 1975) with $\lambda_{\max }$ between $4928 \pm 21 \AA$ (Feb. 4) and $5751 \pm 52 \AA$ (Feb. 18). Position angle is fairly constant in the observed wavelength range for the Asiago spectropolarimetry while a mild trend with wavelength is present in the Crimea data.

\subsection{Time variability}

The variations of the polarization during the outburst are small but significant and they appear to have some connection with the photometric behaviour of the object (Fig. 3). The maximum of polarization during the outburst $(2.92 \%$ in $V$ band) was measured on Feb. 4, two days after the beginning of the rapid rise of luminosity of the object and two days before the visual maximum. One week later, the polarization was $\sim 0.1 \%$ lower while after a further week it stabilized at $\sim 2.5 \%$, with a possible increase of $\sim 0.1-0.2 \%$ during the third peak of the $V$ band light curve (March 2002). The polarization in $V$ band 
Table 2. Polarization of V838 Mon. The integrated $V$ band polarization was computed from the spectra to allow the study of the polarimetric evolution of the object. A and $\mathrm{C}$ refer to Asiago and Crimea observations respectively.

\begin{tabular}{|c|c|c|c|c|c|c|c|c|c|c|}
\hline Date & $P_{U}$ & $\theta_{U}$ & $P_{B}$ & $\theta_{B}$ & $P_{V}$ & $\theta_{V}$ & $P_{R}$ & $\theta_{R}$ & $P_{I}$ & $\theta_{I}$ \\
\hline $10 / 01 / 02 \mathrm{~A}$ & & & & & $2.74 \pm 0.59$ & $159.4 \pm 7.0$ & & & & \\
\hline $11 / 01 / 02 \mathrm{~A}$ & & & & & $2.61 \pm 0.29$ & $159.8 \pm 2.9$ & & & & \\
\hline $04 / 02 / 02 \mathrm{~A}$ & & & & & $2.92 \pm 0.10$ & $160.6 \pm 0.8$ & & & & \\
\hline $11 / 02 / 02 \mathrm{~A}$ & & & & & $2.80 \pm 0.06$ & $157.7 \pm 0.7$ & & & & \\
\hline $11 / 02 / 02 \mathrm{C}$ & $2.30 \pm 0.36$ & $154.6 \pm 4.5$ & $2.68 \pm 0.10$ & $153.7 \pm 1.1$ & $2.66 \pm 0.06$ & $155.1 \pm 0.7$ & $2.56 \pm 0.04$ & $154.7 \pm 0.5$ & $2.39 \pm 0.04$ & $154.6 \pm 0.5$ \\
\hline $15 / 02 / 02 \mathrm{C}$ & $2.12 \pm 0.17$ & $148.0 \pm 2.3$ & $2.41 \pm 0.04$ & $151.8 \pm 0.5$ & $2.36 \pm 0.04$ & $152.3 \pm 0.5$ & $2.28 \pm 0.04$ & $153.3 \pm 0.4$ & $2.09 \pm 0.03$ & $153.0 \pm 0.5$ \\
\hline $16 / 02 / 02 \mathrm{C}$ & $2.29 \pm 0.17$ & $150.2 \pm 2.2$ & $2.50 \pm 0.06$ & $152.3 \pm 0.7$ & $2.47 \pm 0.04$ & $152.7 \pm 0.5$ & $2.33 \pm 0.02$ & $153.0 \pm 0.3$ & $2.09 \pm 0.02$ & $152.8 \pm 0.2$ \\
\hline $18 / 02 / 02 \mathrm{~A}$ & & & & & $2.49 \pm 0.05$ & $159.3 \pm 0.7$ & & & & \\
\hline $18 / 02 / 02 \mathrm{C}$ & $2.43 \pm 0.29$ & $145.5 \pm 3.4$ & $2.30 \pm 0.06$ & $151.1 \pm 0.7$ & $2.43 \pm 0.04$ & $152.9 \pm 0.4$ & $2.31 \pm 0.02$ & $154.4 \pm 0.3$ & $2.11 \pm 0.02$ & $153.3 \pm 0.2$ \\
\hline 04/03/02 A & & & & & $2.58 \pm 0.10$ & $159.5 \pm 1.2$ & & & & \\
\hline 09/03/02 A & & & & & $2.54 \pm 0.18$ & $158.5 \pm 2.2$ & & & & \\
\hline 09/03/02 C & $2.32 \pm 0.23$ & $150.7 \pm 2.8$ & $2.53 \pm 0.06$ & $152.7 \pm 0.7$ & $2.52 \pm 0.04$ & $153.1 \pm 0.4$ & $2.30 \pm 0.02$ & $153.9 \pm 0.3$ & $2.14 \pm 0.02$ & $153.5 \pm 0.3$ \\
\hline 20/03/02 A & & & $2.40 \pm 0.09$ & $160.1 \pm 1.6$ & $2.35 \pm 0.07$ & $158.4 \pm 1.2$ & & & $2.07 \pm 0.09$ & $157.2 \pm 2.1$ \\
\hline $28 / 10 / 02 \mathrm{~A}$ & & & & & $3.09 \pm 0.58$ & $161.6 \pm 6.4$ & & & & \\
\hline $12 / 11 / 02 \mathrm{~A}$ & & & & & $3.05 \pm 0.35$ & $159.7 \pm 3.9$ & & & & \\
\hline
\end{tabular}

in October-November 2002 (not shown in Fig. 3) possibly increases back to higher values $(\sim 3.1 \%)$ but the errors are larger due to the much fainter magnitude of the object $(V=16.05$, Munari et al. 2002b) and therefore we do not speculate any further about it. The polarization contamination due to the patchy nature of the light echo is estimated to be smaller than the photon noise.

We note that the $V$ band flux in October-November 2002 is dominated by the hot B3 component (Munari et al. 2002b), with the cool component contributing $\sim 10 \%$ of the light at most.

\subsection{The interstellar polarization}

The small time variability of the polarization of V838 Mon during and after the outburst, the constancy of the position angle, the polarization in the core of $\mathrm{H}_{\alpha}$ and the wavelength dependence of the polarization, compatible with the Serkowski law, indicate that most of the observed polarization is of interstellar origin (Mc Lean \& Clarke 1979).

To estimate the value of the interstellar polarization we consider the following points:

- the polarization is fairly constant after Feb. 15, 2002, with a possible marginal increase $(0.1 \%)$ during the third peak of the light curve;

- the fit of the Serkowski law as modified by Whittet et al. (1992) to the Feb. 18 spectrum gives $P_{\max }=2.50 \pm 0.05$, $\theta_{\max }=159.0^{\circ}$ at $\lambda_{\max }=5751 \pm 52 \AA$ (Fig. 4). Such a value of $\lambda_{\max }$ is typical for the interstellar medium;

- the residual polarization in the core of $\mathrm{H}_{\alpha}$ on 11th Feb. spectrum is $2.35 \%$ with $\theta=159^{\circ}$. Using the Serkowski law derived on Feb. 18 this gives $P_{\max }=2.46 \pm 0.06$ at $5751 \AA$, fully compatible with the previous estimate.

From these fully consistent estimates we adopt as the interstellar polarization $P_{\max }=2.48 \pm 0.10$ (including zero point uncertainty) with the wavelength dependence obtained from the Feb. 18 spectrum $\left(\lambda_{\max }=5751 \pm 52 \AA\right)^{3}$.

Considering the mentioned systematic discrepancy between Asiago and Crimea data, we adopt as position angle $\theta=156 \pm 3^{\circ}$. In the subtraction of the interstellar polarization from Asiago spectra, we consider instead the position angle of the Asiago dataset $\left(\theta=159^{\circ}\right)$ for consistency.

The contribution of the circumstellar material not directly associated with the outburst, whose presence was shown by the light echo and the IRAS detection of the progenitor (Munari et al. 2002a; Kimeswenger et al. 2002), could add to the fraction of purely interstellar polarization. Hereafter we will refer to interstellar polarization as also including such (possible) circumstellar effects.

\subsection{Intrinsic polarization}

To make more evident the wavelength dependence and the time variability of the intrinsic polarization, we subtracted the interstellar polarization derived in Sect. 4.3 from the observed one (Fig. 6). The maximum of intrinsic polarization is reached on the Feb. 4 spectrum. The intrinsic polarization shows a marked increase toward shorter wavelength, reaching $0.7 \%$ at $5000 \AA$, with position angle $\theta=170^{\circ}$. Polarimetric data on $11 \mathrm{Feb}$. also show the presence of intrinsic polarization, about $0.3 \%$, with a position angle $175 \pm 6^{\circ}$. The wavelength dependence of the intrinsic polarization indicates that further mechanism(s) beside Thomson scattering are at work.

A trend of intrinsic polarization increasing toward shorter wavelength in the range 4500-7500 $\AA$ has been observed in

\footnotetext{
3 The fit of the Serkowski law as modified by Whittet et al. (1992) to the polarimetric data is fully consistent with that from spectropolarimetry concerning the value of polarization $\left(P_{\max }=2.49 \%\right)$ while the $\lambda_{\max }$ is bluer by about $500 \AA$. In spite of the wider wavelength coverage, the wavelength dependence of the interstellar polarization from photometric data is much more uncertain because of the limited number of bands (one of which is affected by large internal errors) and the uncertainty in the effective wavelengths for an object with such a peculiar spectral energy distribution as V838 Mon.
} 


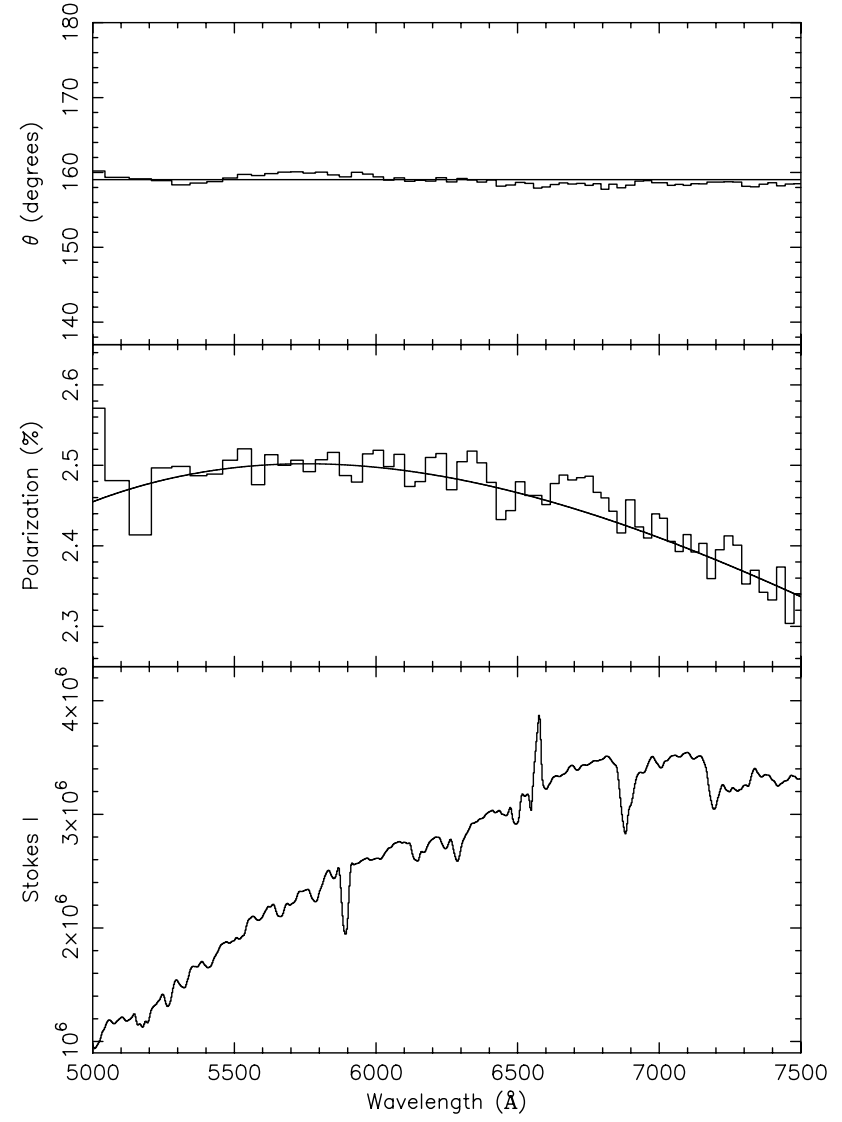

Fig. 4. Position angle, polarization and intensity on 18th Feb. 2002, mainly showing interstellar polarization. The fit of the Serkowski law as modified by Whittet et al. (1992) is overplotted.

several other objects. In the case of the Be star $\zeta$ Tau a complex pattern of polarization as a function of wavelength was observed, with large discontinuities at the Balmer and Paschen edges and a polarization increasing blueward in the Paschen continuum (Wood et al. 1997). This can be explained in terms of electron scattering modified by continuous hydrogen absorptive opacity (Wood et al. 1996). However, our data do not reach the Paschen and Balmer jumps, so that we cannot conclude that the observed trend is due to this effect. A possible Paschen jump is seen in the Wisniewski et al. (2003) data, but only at the one-sigma level.

The observed trend could be ascribed also to a dust disk formed in the stellar outflow (see e.g. Clayton et al. 1997).

In the case of the nova V4444 Sgr (Kawabata et al. 2000), the variation of polarization with wavelength was instead interpreted as Mie scattering due to a preexisting circumstellar dust cloud at fairly large distances from the object. This explanation is probably not correct for V838 Mon since the intrinsic polarization is variable on fairly short timescales.

\subsection{Polarization across spectral lines}

The resolution of the spectra obtained with Grism \#8 enables study of the polarization across spectral lines. For example, V838 Mon developed a prominent $\mathrm{H}_{\alpha}$ emission line during the second maximum.

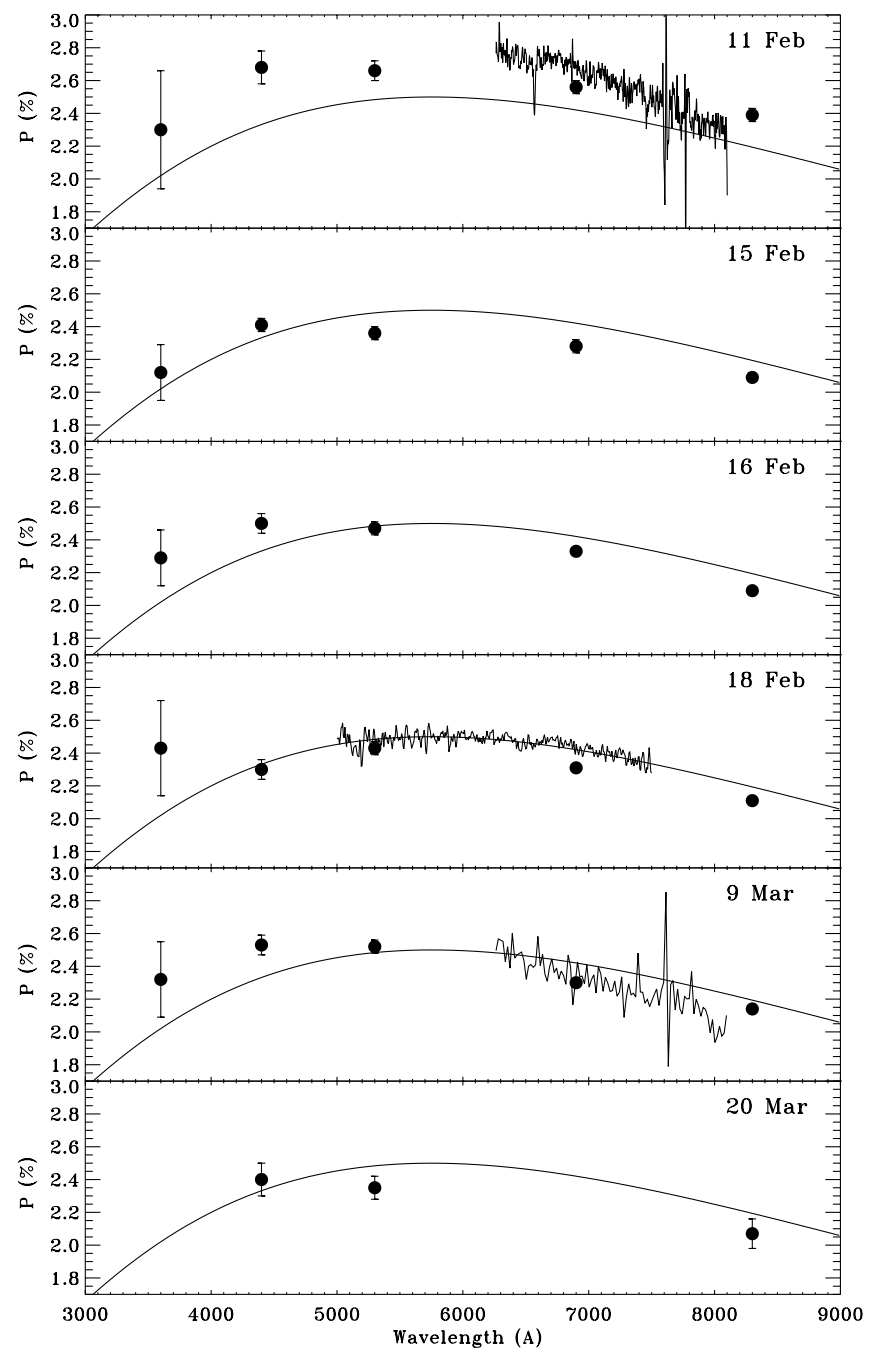

Fig. 5. Polarimetric photometry from Feb. 11 to Mar. 20, 2002. The spectro-polarimetric data taken on Feb. 11, Feb. 18 and Mar. 9 are overplotted on the corresponding panel. The fit of the Serkowski law performed on the spectrum of Feb. 18 is overplotted on each panel. The 11th Feb. data are clearly above the fit, indicating the presence of intrinsic polarization, demonstrated also by the depolarization of the $\mathrm{H}_{\alpha}$ emission line (see below). The other data are in reasonable agreement, indicating that after Feb. 15 the polarization is mainly interstellar. Some discrepancy between broad band and low resolution spectroscopic polarimetry may be due to the very peculiar spectral energy distribution of the object $(B-V>1.5$ at the epochs of polarimetric photometry).

Figure 7 shows the polarization around $\mathrm{H}_{\alpha}$ at three epochs (subtracting the interstellar polarization). A marked depolarization, at $\sim 0.4 \%$ level, is evident in the spectrum of Feb. 11, 5 days after the maximum of the $V$ light curve. A change in the position angle across the line profile is also clearly seen.

A marginal depolarization of $\mathrm{H}_{\alpha}$, possibly characterized by a broader profile, may be present in the Feb. 4 spectrum (two days before the peak of the light curve), when $\mathrm{H}_{\alpha}$ was even brighter but was characterized by a very different profile, without the P-Cyg absorption and very broad wings. No depolarization was detected on Mar. 9, when $\mathrm{H}_{\alpha}$ emission was significantly fainter and the intrinsic polarization of the object was 


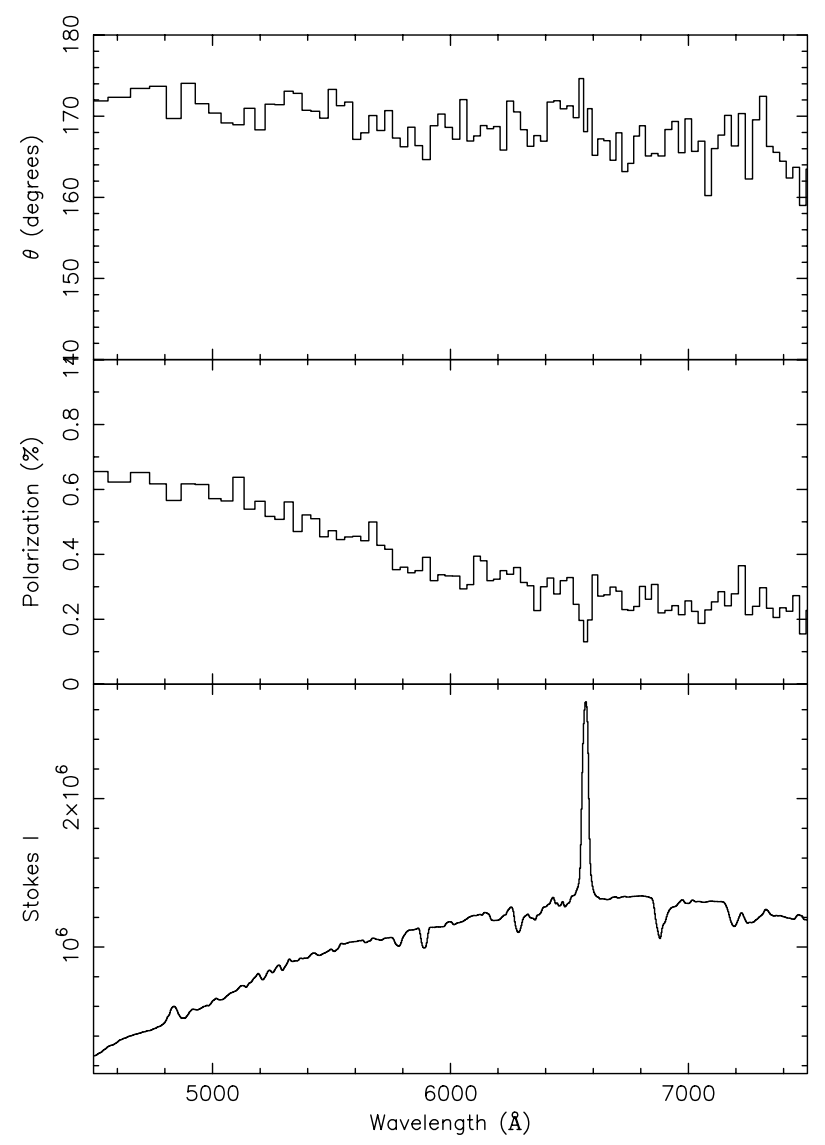

Fig. 6. Intrinsic polarization on Feb. 4, 2002. The insterstellar polarization, taken from the Serkowski fit of Feb. 18, 2002 (see Fig. 4), was subtracted. Note the increasing polarization toward shorter wavelengths and the possible partial depolarization of $\mathrm{H}_{\alpha}$.

small $(\sim 0.1-0.2 \%$ at most). The result of Wisniewski et al. (2003) indicate complete $\mathrm{H}_{\alpha}$ depolarization on 8th Feb.

\subsection{Comparison with Wisniewski et al. (2003)}

Wisniewski et al. (2003) presented spectropolarimetry of V838 Mon obtained in two epochs, Feb. 8 and Feb. 13. On Feb. 8 the $R$-band polarization was $3.2 \%$. The depolarization across the $\mathrm{H}_{\alpha}$ line at this date and the lower polarization of their later spectrum $(P=2.67 \%)$ indicate the presence of intrinsic polarization.

Our data on polarization of V838 Mon agree qualitatively with these results. Our measurements do not reach polarization as high as $3.2 \%$ during the outburst, but this may be due to intrinsic variability of the object and different observing dates. We noted that the polarization seems to follow the photometric evolution (Fig. 3), so that the maximum could have occurred between our observations of Feb. 4 and 11, 2002. The polarization measured by Wisniewski et al. (2003) on Feb. 13 is intermediate between ours on Feb. 11 and 18. Their position angle is $153^{\circ}$, similar to that resulting from Crimea data (see Sect. 4.1).

We note that our data on Feb. 11 reveal the presence of intrinsic polarization at the $\sim 0.3-0.4 \%$ level, so that the assumption by Wisniewski et al. (2003) that the polarization on 13th Feb. was purely interstellar is questionable. Furthermore, a visual inspection of their Fig. 4 reveals that the minimum of polarization in the core of $\mathrm{H}_{\alpha}$ is lower (by $\sim 0.2 \%$ ) than their fit for the interstellar polarization and more compatible with our estimate.

The trend of intrinsic polarization with wavelength of their Feb. 8 spectrum is remarkably similar to our on Feb. 4 in the overlap region. However, their position angle for the intrinsic polarization $\left(\theta=127^{\circ}\right)$ is quite different from our determination.

\subsection{The polarization of stars in the field of V838 Mon}

The study of the polarization of field stars is useful to confirm our estimate of interstellar polarization and it constrains the distance and reddening estimates of the target.

Towards this aim, we measured the polarization of about 40 field stars in the direction of V838 Mon in our deep polarimetric $V$ images taken on October and November 2002. Stars projected inside the light echo were not considered.

Errors are quite large due to the faintness of most of the measured stars; however it appears that about half of the field stars with polarization larger than $0.5 \%$ have position angles similar to that of the estimated interstellar polarization of V838 Mon. This is expected for field stars seen toward coherent portions of interstellar medium. Furthermore most of the stars with such position angle have lower polarization than V838 Mon. All these stars are within 5 arcmin from V838 Mon, so that the spatial inhomogeneities of the interstellar medium should likely play a marginal role. Therefore we can infer that V838 Mon is at larger distance from us than most of the surrounding field stars. However, the lack of distance estimates for these stars does not allow us to quantify this result in terms of a lower limit to the distance. Observational efforts aimed at obtaining their distances using spectral classification are in progress. Details will be presented elsewhere.

\subsection{The polarization of the light echo}

Our deep polarimetric imaging of the field of V838 Mon (Nov. 9, 2002) covers the whole extension of the light echo in the north-south direction and 18 arcsec east-west (Fig. 8).

The light echo shows a distributed polarization with a complex pattern, reaching a $45 \%$ maximum and $88^{\circ}$ position angle at 11 arcsec south and 3 arcsec west of the central star. Typical errors on polarization span from $1.5 \%$ in the brightest zones to $5 \%$ in the faintest. Note that the position angle quoted by Giro et al. (2002) is wrong by $90^{\circ}$.

A large polarization of the light echo was also reported by Bond et al. (2003). On their HST polarimetric images (not fully calibrated), obtained two months earlier than ours (when the light echo appearence was understandably different) they obtained a peak polarization "of $50 \%$ at the inner rim of the cavity, south east of the star". The very different spatial resolution between ground-based and HST observations also plays a role, in addition to changing aspect of the light echo with time, in accounting for the differences between our and the Bond et al. 

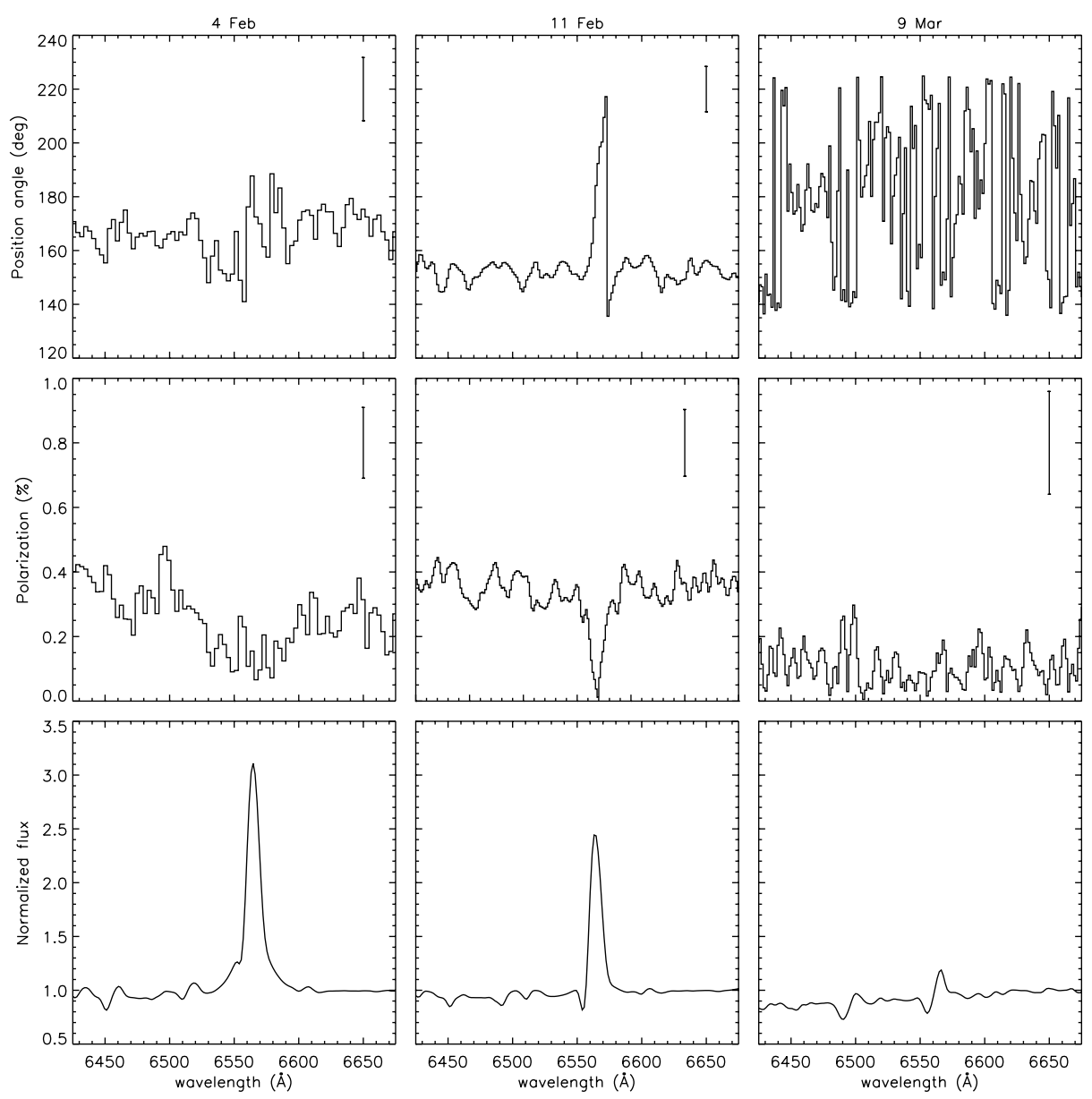

Fig. 7. Polarization across $\mathrm{H}_{\alpha}$ for the spectra obtained with Grism \#8 (from top to bottom) on Feb. 4, Feb. 11 and March 9, 2002. The interstellar polarization, estimated as the polarization in the core of $\mathrm{H}_{\alpha}$ on the 11th Feb. spectrum, was subtracted. Typical errorbars of polarization and position angle are overplotted. The very noisy position angle on 9th March spectrum is the result of the very low intrinsic polarization.

location of peak polarization while maximum values however fairly well agree. Bond et al. (2002) observations on May 20 also indicate that the polarization degree and the position of the maximum evolves with time.

The general appearance is that of a dipole with its axis roughly oriented in the east-west direction and a more visible southern lobe. Such a structure is different to that expected from light scattered by a homogeneous circumstellar medium (see, e.g. Sparks 1994). We also note that about 14 arcsec south of the central object, the polarization degree of the lobe suddenly drops from $\sim 40 \%$ down to $\sim 17 \%$. The northern lobe might possess a quite similar structure as suggested by the fainter features observed close to the star. Due to the limitation of our field of view in the east-west direction, we are unable to assess the geometrical structure of the polarization in the external regions. However, the general constancy of the polarization degree is quite evident.

\section{Clues on reddening and distance}

The distance to V838 Mon is very uncertain, with literature estimates ranging from $650 \mathrm{pc}$ (Kimeswenger et al. 2002) to $10 \mathrm{kpc}$ (Munari et al. 2002b). Reddening is likely constrained in the range $E(B-V) \sim 0.4-0.9$. A further estimate of the reddening can be derived using the limit $E(B-V)>P_{\text {interstellar }} / 9$ (Serkowski et al. 1975). This gives $E(B-V)>0.28$. Adopting the more typical value $E(B-V) \sim P_{\text {interstellar }} / 5$ we get $E(B-$ $V) \sim 0.50$. This is similar to the value $E(B-V)=0.50$ adopted by Munari et al. (2002a) and to $E(B-V)=0.54$ recently found by Wagner \& Starrfield (2002) from the equivalent width of $\mathrm{Na}$ I D lines in the spectrum of the blue component of the system. It is instead smaller than $E(B-V)=0.80$ quoted by Zwitter \& Munari (2002) based on the intensity of $\mathrm{Na} \mathrm{I}$ and $\mathrm{K} \mathrm{I}$ interstellar lines on outburst spectra, $E(B-$ $V)=0.7$ derived by Kimeswenger et al. (2002) by photometric and spectroscopic observations during of the outburst and $E(B-V)=0.9$ estimated by Munari et al. (2002b) from spectral classification and photometry of the hot component. The larger values of reddening, if confirmed, would indicate a ratio $P_{\text {interstellar }} / E(B-V) \sim 3$, that is still fairly normal (see Serkowski et al. 1975).

The wavelength dependence of the interstellar polarization, following a Serkowski law with $\lambda_{\max }=5751 \pm 52 \AA$, implies a normal ratio of total to selective absorption $\left(R_{V}=3.22 \pm 0.17\right.$ using the calibration by Whittet \& van Breda 1978) ${ }^{4}$. This

${ }^{4}$ This is also fully consistent with the dependence of $R_{V}$ on galactic latitude derived by Whittet (1977). 


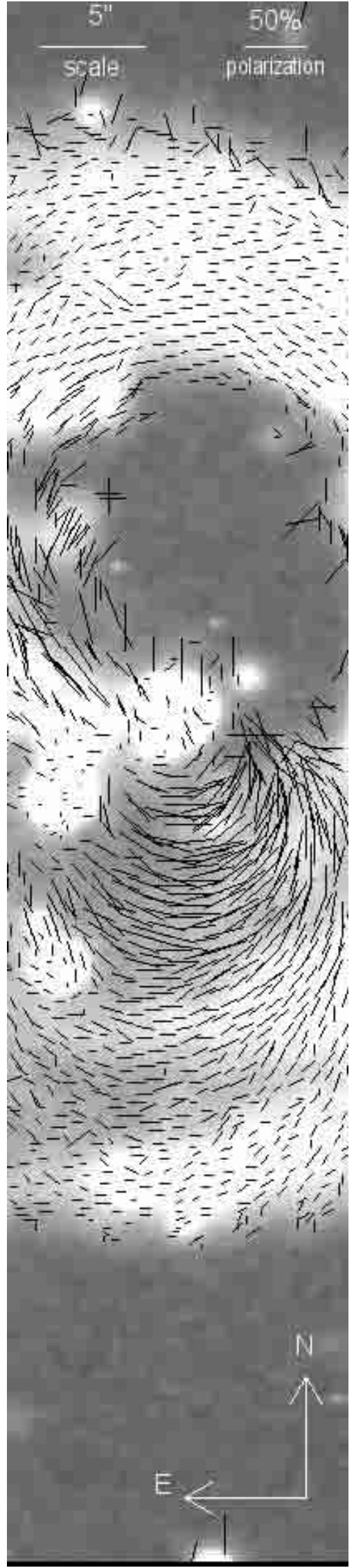

Fig. 8. Polarization map of a portion of the light echo of V838 Monocerotis on Nov. 9, 2002. The central object is V838 Mon. Typical errors on polarization span from $1.5 \%$ in the brightest zones to $5 \%$. leaves little room for the presence of a significant circumstellar absorption characterized by peculiar dust-to-gas ratio or grain composition and nature. The similar polarization angle between V838 Mon and a large fraction of field stars also indicates a minor contribution of the circumstellar material to the observed reddening.

The polarization larger than most of the field stars and the value of reddening likely in the range $0.50-0.80$ point to a fairly large distance, considering that on the reddening maps by Nekel \& Klare (1980) such values of reddening are reached for distances larger than $3 \mathrm{kpc}$. This is in qualitative agreement with the recent results by Bond et al. (2003).

\section{Conclusion}

We have monitored the polarization of V838 Mon from January to November 2002 covering the relevant phases of the evolution of this mysterious object. The main results of this study are:

- The observed polarization of V838 Mon varies between 2.4 and $3.1 \%$.

- Interstellar (+ circumstellar) polarization is $\sim 2.48 \%$ with position angle $\theta=156 \pm 3^{\circ}$. This represents the major contribition to the observed value.

- The intrinsic polarization seems to follow the light curve of V838 Mon, reaching a maximum of $0.7 \%$ at $5000 \AA$ on Feb. 4 , and then quickly declining to zero in $\sim 10$ days. The occurrence of intrinsic polarization could be explained by departures from spherical geometry during the outburst.

- The intrinsic polarization of Feb. 4 shows a marked wavelength dependence, increasing toward shorter wavelengths. This trend is compatible with that expected in the case of electron scattering modified by continuous hydrogen absorptive opacity (Wood et al. 1996), but our limited wavelength coverage does not allow conclusive results to be reached.

- The $\mathrm{H}_{\alpha}$ emission line is depolarized in the spectrum of Feb. 11.

- The analysis of the polarimetric map of the light echo indicates a complex polarization pattern, reaching $P_{\max }=45 \%$.

- The interstellar polarization implies $E(B-V)>0.28$. A higher reddening $E(B-V) \sim 0.5$, in better agreement with independent estimates, is obtained adopting a more typical value for the ratio $P_{\text {interstellar }} / E(B-V)$.

- A normal ratio of total to selective absorption $\left(R_{V}=3.22 \pm 0.17\right)$ is derived from the wavelength of maximum interstellar polarization, suggesting a normal interstellar medium toward V838 Mon.

Acknowledgements. We warmly thank the Asiago Observatory Staff for its support during observations and G. Baume for observing V838 Mon for us during his observing time. We are grateful to V. Piirola for the permission to use his photopolarimeter in these observations. We thank the referee Dr. G. Clayton for stimulating comments. This research made use of the SIMBAD database, operated at CDS, Strasbourg, France. TT acknowledges support of Polish KBN Grant No. 5 P03D 00320. 


\section{References}

Banerjee, D. P. K., \& Ashok, N. M. 2002, A\&A, 395, 161

Bond, H. E., Panagia, N., Sparks, W. B., et al. 2002, IAUC, 7943

Bond, H. E., Henden, A., Levay, Z. G., et al. 2003, Nature, 422, 405

Clayton, G. C., Bjorkman, K. S., Nordsiek, K. H., Zellner, N. E. B., \& Schulte-Ladbeck, R. E. 1997, ApJ, 476, 870

Crause, L. A., Lawson, W. A., Kilkenny, D., et al. 2003, MNRAS, 341,785

Desidera, S., Fantinel, D., \& Giro, E. 2002, AFOSC User Manual, Osservatorio Astronomico di Padova (www.pd.astro.it/Asiago/2000/2300/2310.html)

Desidera, S., \& Munari, U. 2002, IAUC, 7982

Giro, E., Desidera, S., Munari, U., Pernechele, C., \& Baume, G. 2002, IAUC, 8025

Giro, E., Pernechele, C., Munari, U., Boschi, F., \& Tomov, T. 2003, in Symbiotic stars probing stellar evolution, ASP Conf. Ser., 303, ed. R. L. M. Corradi, J. Mikolajewska, \& T. J. Mahoney

Goranskii, V. P., Kusakin, A. V., Metlova, N. V., et al. 2002, Astron. Lett., 28, 691

Henden, A., Munari, U., Marrese, P., Boschi, F., \& Corradi, R. 2002, IAUC, 7859

Kawabata, K. S., Hirata, R., Ikeda, Y., et al. 2000, ApJ, 540, 429

Kimeswenger, S., Lederle, C., Schmeja, S., \& Armsdorfer, B. 2002 MNRAS, 336, 43
Kolev, P., Mikolajewski, M., Tomov, T., et al. 2002 [astro-ph 0211380]

Lorenzi, V. 2003, Master Thesis, University of Padova

Mc Lean, I. S., \& Clarke, D. 1979, MNRAS, 186, 245

Munari, U., Henden, A., Kiyota, S., et al. 2002a, A\&A, 389, L51

Munari, U., Desidera, S., \& Henden, A. 2002b, IAUC, 8005

Nekel, T., \& Klare, G. 1980, A\&AS, 42, 251

Pernechele, C., Giro, E., \& Fantinel, D. 2003, SPIE Proc., 4843, 156

Piirola, V. 1973, A\&A, 27, 383

Piirola, V. 1988, in Polarized radiation of circumstellar origin, ed. G. V. Coyne, A. M. Magalhaes, A. F. J. Moffat, et al., Vatican Observatory - Vatican City State, 735

Serkowski, K., Mathewson, D. S., \& Ford, V. L. 1975, ApJ, 196, 261

Sparks, W. B. 1994, ApJ, 433, 19

Wagner, R. M., \& Starrfield, S. G. 2002, IAUC, 7992

Whittet, D. C. B. 1977, MNRAS, 180, 29

Whittet, D. C. B., \& van Breda, I. G. 1978, A\&A, 66, 57

Whittet, D. C. B., Martin, P. G., Hough, J. H., et al. 1992, ApJ, 386, 562

Wisniewski, J. P., Morrison, N. D., Bjorkman, K. S., et al. 2003, ApJ, 588,486

Wood, K., Bjorkman, J. E., Whitney, B., \& Code, A. 1996, ApJ, 461, 847

Wood, K., Bjorkman, K. S., \& Bjorkman, J. E. 1997, ApJ, 477, 926

Zwitter, T., \& Munari, U. 2002, IAUC, 7812 